CUBO A Mathematical Journal

Vol.17, $N^{\underline{O}} 01$, (65-73). March 2015

\title{
Spline left fractional monotone approximation involving left fractional differential operators
}

\author{
George A. Anastassiou \\ Department of Mathematical Sciences, \\ University of Memphis, \\ Memphis, TN 38152, U.S.A. \\ ganastss@memphis. edu
}

\begin{abstract}
Let $f \in C^{s}([-1,1]), s \in \mathbb{N}$ and $L^{*}$ be a linear left fractional differential operator such that $L^{*}(f) \geq 0$ on $[0,1]$. Then there exists a sequence $Q_{n}, n \in \mathbb{N}$ of polynomial splines with equally spaced knots of given fixed order such that $L^{*}\left(Q_{n}\right) \geq 0$ on $[0,1]$. Furthermore $f$ is approximated with rates fractionally and simultaneously by $Q_{n}$ in the uniform norm. This constrained fractional approximation on $[-1,1]$ is given via inequalities invoving a higher modulus of smoothness of $f^{(s)}$.
\end{abstract}

\section{RESUMEN}

Sea $f \in C^{s}([-1,1]), s \in \mathbb{N}$ y $L^{*}$ un operador diferencial fraccionario lineal izquierdo tal que $L^{*}(f) \geq 0$ en $[0,1]$.. Entonces, existe una sucesión $Q_{n}, n \in \mathbb{N}$ de splines polinomiales con nodos equiespaciados de un orden fijo dado tal que $L^{*}\left(Q_{n}\right) \geq 0$ en $[0,1]$. Además, $f$ se aproxima con velocidades fraccionales y simultáneamente por $Q_{n}$ en la norma uniforme. Esta aproximación fraccional restringida a $[-1,1]$ se encuentra por medio de desigualdades que involucran un módulo alto de suavidad de $\mathrm{f}^{(\mathrm{s})}$.

Keywords and Phrases: Monotone Approximation, Caputo fractional derivative, fractional linear differential operator, modulus of smoothness, splines.

2010 AMS Mathematics Subject Classification: 26A33, 41A15, 41A17, 41A25, 41A28, 41A29, 41A99. 


\section{Introduction}

Let $[a, b] \subset \mathbb{R}$ and for $n \geq 1$ consider the partition $\Delta_{n}$ with points $x_{i n}=a+i\left(\frac{b-a}{n}\right)$, $i=0,1, \ldots, n$. Hence $\bar{\Delta}_{n} \equiv \max _{1 \leq i \leq n}\left(x_{i n}-x_{i-1, n}\right)=\frac{b-a}{n}$.

Let $S_{m}\left(\Delta_{n}\right)$ be the space of polynomial splines of order $m>0$ with simple knots at the points $x_{i n}, i=1, \ldots, n-1$. Then there exists a linear operator $Q_{n}: Q_{n} \equiv Q_{n}(f)$, mapping $B[a, b]$ : the space of bounded real valued functions $f$ on $[a, b]$, into $S_{m}\left(\Delta_{n}\right)$ (see [4, p. 224, Theorem 6.18).

From the same reference 4, p. 227, Corollary 6.21, we get

Corolary 1. Let $1 \leq \sigma \leq \mathrm{m}, \mathrm{n} \geq 1$. Then for all $\mathrm{f} \in \mathrm{C}^{\sigma-1}[\mathrm{a}, \mathrm{b}] ; \mathrm{r}=0, \ldots, \sigma-1$,

$$
\left\|f^{(r)}-Q_{n}^{(r)}\right\|_{\infty} \leq C_{1}\left(\frac{b-a}{n}\right)^{\sigma-r-1} \omega_{m-\sigma+1}\left(f^{(\sigma-1)}, \frac{b-a}{n}\right),
$$

where $\mathrm{C}_{1}$ depends only on $\mathrm{m}, \mathrm{C}_{1}=\mathrm{C}_{1}(\mathrm{~m})$.

By denoting $C_{2}=C_{1} \max _{0 \leq r \leq \sigma-1}(b-a)^{\sigma-r-1}$ we obtain

Lemma 1. ([1]) Let $1 \leq \sigma \leq \mathrm{m}, \mathrm{n} \geq 1$. Then for all $\mathrm{f} \in \mathrm{C}^{\sigma-1}[\mathrm{a}, \mathrm{b}] ; \mathrm{r}=0, \ldots, \sigma-1$,

$$
\left\|f^{(r)}-Q_{n}^{(r)}\right\|_{\infty} \leq \frac{C_{2}}{n^{\sigma-r-1}} \omega_{m-\sigma+1}\left(f^{(\sigma-1)}, \frac{b-a}{n}\right),
$$

where $\mathrm{C}_{2}$ depends only on $\mathrm{m}, \sigma$ and $\mathrm{b}-\mathrm{a}$. Here $\omega_{\mathrm{m}-\sigma+1}$ is the usual modulus of smoothness of order $\mathrm{m}-\sigma+1$.

We are motivated by

Theorem 1. ([1]) Let $\mathrm{h}, \mathrm{k}, \sigma, \mathrm{m}$ be integers, $0 \leq \mathrm{h} \leq \mathrm{k} \leq \sigma-1, \sigma \leq \mathrm{m}$ and let $\mathrm{f} \in \mathrm{C}^{\sigma-1}[\mathrm{a}, \mathrm{b}]$. Let $\alpha_{j}(x) \in B[a, b], j=h, h+1, \ldots, k$ and suppose that $\alpha_{h}(x) \geq \alpha>0$ or $\alpha_{h}(x) \leq \beta<0$ for all $x \in[a, b]$. Take the linear differential operator

$$
L=\sum_{j=h}^{k} \alpha_{j}(x)\left[\frac{d^{j}}{d x^{j}}\right]
$$

and assume, throughout $[\mathrm{a}, \mathrm{b}]$,

$$
L(f) \geq 0 .
$$

Then, for every integer $\mathrm{n} \geq 1$, there is a polynomial spline function $\mathrm{Q}_{\mathrm{n}}(\mathrm{x})$ of order $\mathrm{m}$ with simple knots at $\left\{\mathrm{a}+\mathrm{i}\left(\frac{\mathrm{b}-\mathrm{a}}{\mathrm{n}}\right), \mathrm{i}=1, \ldots, \mathrm{n}-1\right\}$ such that $\mathrm{L}\left(\mathrm{Q}_{\mathrm{n}}\right) \geq 0$ throughout $[\mathrm{a}, \mathrm{b}]$ and

$$
\left\|f^{(r)}-Q_{n}^{(r)}\right\|_{\infty} \leq \frac{C}{n^{\sigma-k-1}} \omega_{m-\sigma+1}\left(f^{(\sigma-1)}, \frac{b-a}{n}\right), \quad 0 \leq r \leq h .
$$


Moreover, we find

$$
\left\|f^{(r)}-Q_{n}^{(r)}\right\|_{\infty} \leq \frac{C}{n^{\sigma-r-1}} \omega_{m-\sigma+1}\left(f^{(\sigma-1)}, \frac{b-a}{n}\right), \quad h+1 \leq r \leq \sigma-1,
$$

where $\mathrm{C}$ is a constant independent of $\mathrm{f}$ and $\mathrm{n}$. It depends only on $\mathrm{m}, \sigma, \mathrm{L}, \mathrm{a}, \mathrm{b}$.

Next we specialize on the case of $a=-1, b=1$. That is working on $[-1,1]$.

By Lemma 1 we get

Lemma 2. Let $1 \leq \sigma \leq \mathrm{m}, \mathrm{n} \geq 1$. Then for all $\mathrm{f} \in \mathrm{C}^{\sigma-1}([-1,1]) ; \mathrm{j}=0,1, \ldots, \sigma-1$,

$$
\left\|f^{(j)}-Q_{n}^{(j)}\right\|_{\infty} \leq \frac{C_{2}}{n^{\sigma-j-1}} \omega_{m-\sigma+1}\left(f^{(\sigma-1)}, \frac{2}{n}\right),
$$

where $\mathrm{C}_{2}:=\mathrm{C}_{2}(\mathrm{~m}, \sigma):=\mathrm{C}_{1}(\mathrm{~m}) 2^{\sigma-1}$.

Since

$$
\omega_{m-\sigma+1}\left(f^{(\sigma-1)}, \frac{2}{n}\right) \leq 2^{m-\sigma+1} \omega_{m-\sigma+1}\left(f^{(\sigma-1)}, \frac{1}{n}\right)
$$

(see 2], p. 45), we get

Lemma 3. Let $1 \leq \sigma \leq \mathrm{m}, \mathrm{n} \geq 1$. Then for all $\mathrm{f} \in \mathrm{C}^{\sigma-1}([-1,1]) ; \mathrm{j}=0,1, \ldots, \sigma-1$,

$$
\left\|f^{(j)}-Q_{n}^{(j)}\right\|_{\infty} \leq \frac{C_{2}^{*}}{n^{\sigma-j-1}} \omega_{m-\sigma+1}\left(f^{(\sigma-1)}, \frac{1}{n}\right)
$$

where $C_{2}^{*}:=C_{2}^{*}(m, \sigma):=C_{1}(m) 2^{m}$.

We use a lot in this article Lemma 3 ,

In this article we extend Theorem 1 over $[-1,1]$ to the fractional level. Indeed here $\mathrm{L}$ is replaced by $L^{*}$, a linear left Caputo fractional differential operator. Now the monotonicity property is only true on the critical interval $[0,1]$. Simultaneous fractional convergence remains true on all of $[-1,1]$.

We make

Definition 1. ([3], p. 50) Let $\alpha>0$ and $\lceil\alpha\rceil=\mathrm{m}$, ( $\lceil\cdot\rceil$ ceiling of the number). Consider $f \in \mathrm{C}^{\mathrm{m}}([-1,1])$. We define the left Caputo fractional derivative of $\mathrm{f}$ of order $\alpha$ as follows:

$$
\left(D_{*-1}^{\alpha} f\right)(x)=\frac{1}{\Gamma(m-\alpha)} \int_{-1}^{x}(x-t)^{m-\alpha-1} f^{(m)}(t) d t
$$

for any $x \in[-1,1]$, where $\Gamma$ is the gamma function.

We set

$$
\begin{gathered}
D_{*-1}^{\circ} f(x)=f(x), \\
D_{*-1}^{m} f(x)=f^{(m)}(x), \quad \forall x \in[-1,1] .
\end{gathered}
$$




\section{Main Result}

Theorem 2. Let $\mathrm{h}, \mathrm{k}, \sigma, \mathrm{m}$ be integers, $1 \leq \sigma \leq \mathrm{m}, \mathrm{n} \in \mathbb{N}$, with $0 \leq \mathrm{h} \leq \mathrm{k} \leq \sigma-2$ and let $f \in C^{\sigma-1}([-1,1])$, with $f^{(\sigma-1)}$ having modulus of smoothness $\omega_{m-\sigma+1}\left(f^{(\sigma-1)}, \delta\right)$ there, $\delta>0$. Let $\alpha_{j}(x), j=h, h+1, \ldots, k$ be real functions, defined and bounded on $[-1,1]$ and suppose $\alpha_{h}(x)$ is either $\geq \alpha>0$ or $\leq \beta<0$ on $[0,1]$. Let the real numbers $\alpha_{0}=0<\alpha_{1} \leq 1<\alpha_{2} \leq 2<\ldots<\alpha_{\sigma-2} \leq \sigma-2$. Here $\mathrm{D}_{*-1}^{\alpha_{j}} \mathrm{f}$ stands for the left Caputo fractional derivative of $\mathrm{f}$ of order $\alpha_{j}$ anchored at -1 . Consider the linear left fractional differential operator

$$
L^{*}:=\sum_{j=h}^{k} \alpha_{j}(x)\left[D_{*-1}^{\alpha_{j}}\right]
$$

and suppose, throughout $[0,1], \mathrm{L}^{*}(\mathrm{f}) \geq 0$.

Then, for every integer $\mathrm{n} \geq 1$, there exists a polynomial spline function $\mathrm{Q}_{\mathrm{n}}(\mathrm{x})$ of order $\mathrm{m}>0$ with simple knots at $\left\{-1+i \frac{2}{n}, i=1, \ldots, n-1\right\}$ such that $L^{*}\left(Q_{n}\right) \geq 0$ throughout $[0,1]$, and

$$
\begin{gathered}
\sup _{-1 \leq x \leq 1}\left|\left(D_{*-1}^{\alpha_{j}} f\right)(x)-\left(D_{*-1}^{\alpha_{j}} Q_{n}\right)(x)\right| \leq \\
\frac{2^{j-\alpha_{j}}}{\Gamma\left(j-\alpha_{j}+1\right)} \frac{C_{2}^{*}}{n^{\sigma-j-1}} \omega_{m-\sigma+1}\left(f^{(\sigma-1)}, \frac{1}{n}\right),
\end{gathered}
$$

$j=h+1, \ldots, \sigma-2$.

Set

$$
l_{j}: \equiv \sup _{x \in[-1,1]}\left|\alpha_{h}^{-1}(x) \alpha_{j}(x)\right|, \quad h \leq j \leq k .
$$

When $\mathbf{j}=1, \ldots, \mathrm{h}$ we derive

$$
\begin{gathered}
\max _{-1 \leq x \leq 1}\left|\left(D_{*-1}^{\alpha_{j}} f\right)(x)-\left(D_{*-1}^{\alpha_{j}} Q_{n}\right)(x)\right| \leq \frac{C_{2}^{*}}{n^{\sigma-k-1}} \omega_{m-\sigma+1}\left(f^{(\sigma-1)}, \frac{1}{n}\right) . \\
{\left[\left(\sum_{\tau=h}^{k} l_{\tau} \frac{2^{\tau-\alpha_{\tau}}}{\Gamma\left(\tau-\alpha_{\tau}+1\right)}\right)\left(\sum_{\lambda=0}^{h-j} \frac{2^{h-\alpha_{j}-\lambda}}{\lambda ! \Gamma\left(h-\alpha_{j}-\lambda+1\right)}\right)+\frac{2^{j-\alpha_{j}}}{\Gamma\left(j-\alpha_{j}+1\right)}\right] .}
\end{gathered}
$$

Finally it holds

$$
\begin{gathered}
\sup _{-1 \leq x \leq 1}\left|f(x)-Q_{n}(x)\right| \leq \\
\frac{C_{2}^{*}}{n^{\sigma-k-1}} \omega_{m-\sigma+1}\left(f^{(\sigma-1)}, \frac{1}{n}\right)\left[\frac{1}{h !} \sum_{\tau=h}^{k} l_{\tau} \frac{2^{\tau-\alpha_{\tau}}}{\Gamma\left(\tau-\alpha_{\tau}+1\right)}+1\right] .
\end{gathered}
$$

Proof. Set $\alpha_{0}=0$, thus $\left\lceil\alpha_{0}\right\rceil=0$. We have $\left\lceil\alpha_{j}\right\rceil=j, j=1, \ldots, \sigma-2$.

Let $Q_{n}$ as in Lemma 5. 
We notice that $(x \in[-1,1])$

$$
\begin{gathered}
\left|\left(D_{*-1}^{\alpha_{j}} f\right)(x)-\left(D_{*-1}^{\alpha_{j}} Q_{n}\right)(x)\right|= \\
\frac{1}{\Gamma\left(j-\alpha_{j}\right)}\left|\int_{-1}^{x}(x-t)^{j-\alpha_{j}-1} f^{(j)}(t) d t-\int_{-1}^{x}(x-t)^{j-\alpha_{j}-1} Q_{n}^{(j)}(t) d t\right|= \\
\frac{1}{\Gamma\left(j-\alpha_{j}\right)}\left|\int_{-1}^{x}(x-t)^{j-\alpha_{j}-1}\left(f^{(j)}(t)-Q_{n}^{(j)}(t)\right) d t\right| \leq \\
\frac{1}{\Gamma\left(j-\alpha_{j}\right)} \int_{-1}^{x}(x-t)^{j-\alpha_{j}-1}\left|f^{(j)}(t)-Q_{n}^{(j)}(t)\right| d t \stackrel{9}{\leq} \\
\frac{1}{\Gamma\left(j-\alpha_{j}\right)}\left(\int_{-1}^{x}(x-t)^{j-\alpha_{j}-1} d t\right) \frac{C_{2}^{*}}{n^{\sigma-j-1}} \omega_{m-\sigma+1}\left(f^{(\sigma-1)}, \frac{1}{n}\right)= \\
\frac{1}{\Gamma\left(j-\alpha_{j}\right)} \frac{(x+1)^{j-\alpha_{j}}}{\left(j-\alpha_{j}\right)} \frac{C_{2}^{*}}{n^{\sigma-j-1}} \omega_{m-\sigma+1}\left(f^{(\sigma-1)}, \frac{1}{n}\right)= \\
\frac{(x+1)^{j-\alpha_{j}}}{\Gamma\left(j-\alpha_{j}+1\right)} \frac{C_{2}^{*}}{n^{\sigma-j-1}} \omega_{m-\sigma+1}\left(f^{(\sigma-1)}, \frac{1}{n}\right) \leq \\
\frac{2^{j-\alpha_{j}}}{\Gamma\left(j-\alpha_{j}+1\right)} \frac{C_{2}^{*}}{n^{\sigma-j-1}} \omega_{m-\sigma+1}\left(f^{(\sigma-1)}, \frac{1}{n}\right) .
\end{gathered}
$$

Hence

$$
\left\|D_{*-1}^{\alpha_{j}} f-D_{*-1}^{\alpha_{j}} Q_{n}\right\|_{\infty,[-1,1]} \leq \frac{2^{j-\alpha_{j}}}{\Gamma\left(j-\alpha_{j}+1\right)} \frac{C_{2}^{*}}{n^{\sigma-j-1}} \omega_{m-\sigma+1}\left(f^{(\sigma-1)}, \frac{1}{n}\right),
$$

$j=0,1, \ldots, \sigma-2$.

We set

$$
\rho_{n}:=C_{2}^{*} \omega_{m-\sigma+1}\left(f^{(\sigma-1)}, \frac{1}{n}\right)\left(\sum_{j=h}^{k} l_{j} \frac{2^{j-\alpha_{j}}}{\Gamma\left(j-\alpha_{j}+1\right) n^{\sigma-j-1}}\right) .
$$

I. Suppose, throughout $[0,1], \alpha_{h}(x) \geq \alpha>0$. Let $Q_{n}(x), x \in[-1,1]$, the polynomial spline of order $m>0$ with simple knots at the points $x_{i n}, i=1, \ldots, n-1$, on $[-1,1]\left(x_{i n}=-1+i \frac{2}{n}\right.$, $i=0,1, \ldots, n$, here $\left.\bar{\Delta}_{n}=\frac{2}{n}\right)$, so that

$$
\begin{gathered}
\max _{-1 \leq x \leq 1}\left|D_{*-1}^{\alpha_{j}}\left(f(x)+\rho_{n} \frac{x^{h}}{h !}\right)-\left(D_{*-1}^{\alpha_{j}} Q_{n}\right)(x)\right| \leq \\
\frac{2^{j-\alpha_{j}}}{\Gamma\left(j-\alpha_{j}+1\right)} \frac{C_{2}^{*}}{n^{\sigma-j-1}} \omega_{m-\sigma+1}\left(f^{(\sigma-1)}, \frac{1}{n}\right),
\end{gathered}
$$

$j=0,1, \ldots, \sigma-2$.

When $j=h+1, \ldots, \sigma-2$, then

$$
\max _{-1 \leq x \leq 1}\left|\left(D_{*-1}^{\alpha_{j}} f\right)(x)-\left(D_{*-1}^{\alpha_{j}} Q_{n}\right)(x)\right| \leq
$$




$$
\frac{2^{j-\alpha_{j}}}{\Gamma\left(j-\alpha_{j}+1\right)} \frac{C_{2}^{*}}{n^{\sigma-j-1}} \omega_{m-\sigma+1}\left(f^{(\sigma-1)}, \frac{1}{n}\right),
$$

proving (13).

For $j=1, \ldots, h$ we find that

$$
D_{*-1}^{\alpha_{j}}\left(\frac{x^{h}}{h !}\right)=\sum_{\lambda=0}^{h-j} \frac{(-1)^{\lambda}(x+1)^{h-\alpha_{j}-\lambda}}{\lambda ! \Gamma\left(h-\alpha_{j}-\lambda+1\right)} .
$$

Therefore we get from (23)

$$
\begin{gathered}
\max _{-1 \leq x \leq 1}\left|\left(D_{*-1}^{\alpha_{j}} f\right)(x)+\rho_{n}\left(\sum_{\lambda=0}^{h-j} \frac{(-1)^{\lambda}(x+1)^{h-\alpha_{j}-\lambda}}{\lambda ! \Gamma\left(h-\alpha_{j}-\lambda+1\right)}\right)-\left(D_{*-1}^{\alpha_{j}} Q_{n}\right)(x)\right| \leq \\
\frac{2^{j-\alpha_{j}}}{\Gamma\left(j-\alpha_{j}+1\right)} \frac{C_{2}^{*}}{n^{\sigma-j-1}} \omega_{m-\sigma+1}\left(f^{(\sigma-1)}, \frac{1}{n}\right),
\end{gathered}
$$

$j=1, \ldots, h$.

Therefore we get for $j=1, \ldots, h$, that

$$
\begin{aligned}
& \max _{-1 \leq x \leq 1}\left|\left(D_{*-1}^{\alpha_{j}} f\right)(x)-\left(D_{*-1}^{\alpha_{j}} Q_{n}\right)(x)\right| \leq \\
& \rho_{n}\left(\sum_{\lambda=0}^{h-j} \frac{2^{h-\alpha_{j}-\lambda}}{\lambda ! \Gamma\left(h-\alpha_{j}-\lambda+1\right)}\right)+\frac{2^{j-\alpha_{j}}}{\Gamma\left(j-\alpha_{j}+1\right)} \frac{C_{2}^{*}}{n^{\sigma-j-1}} \omega_{m-\sigma+1}\left(f^{(\sigma-1)}, \frac{1}{n}\right)= \\
& C_{2}^{*} \omega_{m-\sigma+1}\left(f^{(\sigma-1)}, \frac{1}{n}\right)\left(\sum_{\bar{j}=h}^{k} l_{\bar{j}} \frac{2^{\bar{j}-\alpha_{\bar{j}}}}{\Gamma\left(\bar{j}-\alpha_{\bar{j}}+1\right) n^{\sigma-\bar{j}-1}}\right) . \\
& \left(\sum_{\lambda=0}^{h-j} \frac{2^{h-\alpha_{j}-\lambda}}{\lambda ! \Gamma\left(h-\alpha_{j}-\lambda+1\right)}\right)+\frac{2^{j-\alpha_{j}}}{\Gamma\left(j-\alpha_{j}+1\right)} \frac{C_{2}^{*}}{n^{\sigma-j-1}} \omega_{m-\sigma+1}\left(f^{(\sigma-1)}, \frac{1}{n}\right)= \\
& C_{2}^{*} \omega_{m-\sigma+1}\left(f^{(\sigma-1)}, \frac{1}{n}\right)\left[\left(\sum_{\bar{j}=h}^{k} l_{\bar{j}} \frac{2^{\bar{j}-\alpha_{\bar{j}}}}{\Gamma\left(\bar{j}-\alpha_{\bar{j}}+1\right)} \frac{1}{n^{\sigma-\bar{j}-1}}\right) .\right. \\
& \left.\left(\sum_{\lambda=0}^{h-j} \frac{2^{h-\alpha_{j}-\lambda}}{\lambda ! \Gamma\left(h-\alpha_{j}-\lambda+1\right)}\right)+\frac{2^{j-\alpha_{j}}}{\Gamma\left(j-\alpha_{j}+1\right)} \frac{1}{n^{\sigma-j-1}}\right] \leq \\
& C_{2}^{*} \omega_{m-\sigma+1}\left(f^{(\sigma-1)}, \frac{1}{n}\right) \frac{1}{n^{\sigma-k-1}}\left[\left(\sum_{\bar{j}=h}^{k} l_{\bar{j}} \frac{2^{\bar{j}-\alpha_{\bar{j}}}}{\Gamma\left(\bar{j}-\alpha_{\bar{j}}+1\right)}\right) .\right. \\
& \left.\left(\sum_{\lambda=0}^{h-j} \frac{2^{h-\alpha_{j}-\lambda}}{\lambda ! \Gamma\left(h-\alpha_{j}-\lambda+1\right)}\right)+\frac{2^{j-\alpha_{j}}}{\Gamma\left(j-\alpha_{j}+1\right)}\right] \text {. }
\end{aligned}
$$


Hence for $j=1, \ldots, h$ we derived (15):

$$
\begin{aligned}
& \max _{-1 \leq x \leq 1}\left|\left(D_{*-1}^{\alpha_{j}} f\right)(x)-\left(D_{*-1}^{\alpha_{j}} Q_{n}\right)(x)\right| \leq \frac{C_{2}^{*}}{n^{\sigma-k-1}} \omega_{m-\sigma+1}\left(f^{(\sigma-1)}, \frac{1}{n}\right) . \\
& {\left[\left(\sum_{\tau=h}^{k} l_{\tau} \frac{2^{\tau-\alpha_{\tau}}}{\Gamma\left(\tau-\alpha_{\tau}+1\right)}\right)\left(\sum_{\lambda=0}^{h-j} \frac{2^{h-\alpha_{j}-\lambda}}{\lambda ! \Gamma\left(h-\alpha_{j}-\lambda+1\right)}\right)+\frac{2^{j-\alpha_{j}}}{\Gamma\left(j-\alpha_{j}+1\right)}\right] .}
\end{aligned}
$$

When $j=0$ from (23) we obtain

$$
\max _{-1 \leq x \leq 1}\left|f(x)+\rho_{n} \frac{x^{h}}{h !}-Q_{n}(x)\right| \leq \frac{C_{2}^{*}}{n^{\sigma-1}} \omega_{m-\sigma+1}\left(f^{(\sigma-1)}, \frac{1}{n}\right) .
$$

And

$$
\begin{gathered}
\max _{-1 \leq x \leq 1}\left|f(x)-Q_{n}(x)\right| \leq \frac{\rho_{n}}{h !}+\frac{C_{2}^{*}}{n^{\sigma-1}} \omega_{m-\sigma+1}\left(f^{(\sigma-1)}, \frac{1}{n}\right)= \\
\frac{C_{2}^{*}}{h !} \omega_{m-\sigma+1}\left(f^{(\sigma-1)}, \frac{1}{n}\right)\left(\sum_{\tau=h}^{k} l_{\tau} \frac{2^{\tau-\alpha_{\tau}}}{\Gamma\left(\tau-\alpha_{\tau}+1\right) n^{\sigma-\tau-1}}\right) \\
+\frac{C_{2}^{*}}{n^{\sigma-1}} \omega_{m-\sigma+1}\left(f^{(\sigma-1)}, \frac{1}{n}\right)= \\
C_{2}^{*} \omega_{m-\sigma+1}\left(f^{(\sigma-1)}, \frac{1}{n}\right)\left[\frac{1}{h !} \sum_{\tau=h}^{k} l_{\tau} \frac{2^{\tau-\alpha_{\tau}}}{\Gamma\left(\tau-\alpha_{\tau}+1\right) n^{\sigma-\tau-1}}+\frac{1}{n^{\sigma-1}}\right] \leq \\
\frac{C_{2}^{*}}{n^{\sigma-k-1}} \omega_{m-\sigma+1}\left(f^{(\sigma-1)}, \frac{1}{n}\right)\left[\frac{1}{h !} \sum_{\tau=h}^{k} l_{\tau} \frac{2^{\tau-\alpha_{\tau}}}{\Gamma\left(\tau-\alpha_{\tau}+1\right)}+1\right] .
\end{gathered}
$$

Proving

$$
\begin{gathered}
\max _{-1 \leq x \leq 1}\left|f(x)-Q_{n}(x)\right| \leq \\
\frac{C_{2}^{*}}{n^{\sigma-k-1}} \omega_{m-\sigma+1}\left(f^{(\sigma-1)}, \frac{1}{n}\right)\left[\frac{1}{h !} \sum_{\tau=h}^{k} l_{\tau} \frac{2^{\tau-\alpha_{\tau}}}{\Gamma\left(\tau-\alpha_{\tau}+1\right)}+1\right],
\end{gathered}
$$

So that (16) is established.

Also if $0 \leq x \leq 1$, then

$$
\begin{gathered}
\alpha_{h}^{-1}(x) L^{*}\left(Q_{n}(x)\right)=\alpha_{h}^{-1}(x) L^{*}(f(x))+\rho_{n} \frac{(x+1)^{h-\alpha_{h}}}{\Gamma\left(h-\alpha_{h}+1\right)}+ \\
\sum_{j=h}^{k} \alpha_{h}^{-1}(x) \alpha_{j}(x)\left[D_{*-1}^{\alpha_{j}} Q_{n}(x)-D_{*-1}^{\alpha_{j}} f(x)-\frac{\rho_{n}}{h !} D_{*-1}^{\alpha_{j}} x^{h}\right] \stackrel{\sqrt[23]{\geq}}{\geq} \\
\rho_{n} \frac{(x+1)^{h-\alpha_{h}}}{\Gamma\left(h-\alpha_{h}+1\right)}-\left(\sum_{j=h}^{k} l_{j} \frac{2^{j-\alpha_{j}}}{\Gamma\left(j-\alpha_{j}+1\right)} \frac{C_{2}^{*}}{n^{\sigma-j-1}} \omega_{m-\sigma+1}\left(f^{(\sigma-1)}, \frac{1}{n}\right)\right)=
\end{gathered}
$$




$$
\begin{gathered}
\rho_{n} \frac{(x+1)^{h-\alpha_{h}}}{\Gamma\left(h-\alpha_{h}+1\right)}-\rho_{n}=\rho_{n}\left[\frac{(x+1)^{h-\alpha_{h}}}{\Gamma\left(h-\alpha_{h}+1\right)}-1\right]= \\
\rho_{n}\left[\frac{(x+1)^{h-\alpha_{h}}-\Gamma\left(h-\alpha_{h}+1\right)}{\Gamma\left(h-\alpha_{h}+1\right)}\right] \geq \rho_{n}\left[\frac{1-\Gamma\left(h-\alpha_{h}+1\right)}{\Gamma\left(h-\alpha_{h}+1\right)}\right] \geq 0 .
\end{gathered}
$$

Explanation: We know that $\Gamma(1)=1, \Gamma(2)=1$, and $\Gamma$ is convex and positive on $(0, \infty)$. Here $0 \leq h-\alpha_{h}<1$ and $1 \leq h-\alpha_{h}+1<2$. Thus $\Gamma\left(h-\alpha_{h}+1\right) \leq 1$ and $1-\Gamma\left(h-\alpha_{h}+1\right) \geq 0$. Hence $L^{*}\left(Q_{n}(x)\right) \geq 0, x \in[0,1]$.

II. Suppose on $[0,1]$ that $\alpha_{h}(x) \leq \beta<0$. Let $Q_{n}(x), x \in[-1,1]$, be the polynomial spline of order $m>0$, (as before), so that

$$
\begin{gathered}
\max _{-1 \leq x \leq 1}\left|D_{*-1}^{\alpha_{j}}\left(f(x)-\rho_{n} \frac{x^{h}}{h !}\right)-\left(D_{*-1}^{\alpha_{j}} Q_{n}\right)(x)\right| \leq \\
\frac{2^{j-\alpha_{j}}}{\Gamma\left(j-\alpha_{j}+1\right)} \frac{C_{2}^{*}}{n^{\sigma-j-1}} \omega_{m-\sigma+1}\left(f^{(\sigma-1)}, \frac{1}{n}\right),
\end{gathered}
$$

$j=0,1, \ldots, \sigma-2$.

Similarly as before we obtain again inequalities of convergence (13), (15) and (16).

Also if $0 \leq x \leq 1$, then

$$
\begin{gathered}
\alpha_{h}^{-1}(x) L^{*}\left(Q_{n}(x)\right)=\alpha_{h}^{-1}(x) L^{*}(f(x))-\rho_{n} \frac{(x+1)^{h-\alpha_{h}}}{\Gamma\left(h-\alpha_{h}+1\right)}+ \\
\sum_{j=h}^{k} \alpha_{h}^{-1}(x) \alpha_{j}(x)\left[D_{*-1}^{\alpha_{j}} Q_{n}(x)-D_{*-1}^{\alpha_{j}} f(x)+\frac{\rho_{n}}{h !}\left(D_{*-1}^{\alpha_{j}} x^{h}\right)\right] \stackrel{\text { 377 }}{\leq} \\
-\rho_{n} \frac{(x+1)^{h-\alpha_{h}}}{\Gamma\left(h-\alpha_{h}+1\right)}+\sum_{j=h}^{k} l_{j} \frac{2^{j-\alpha_{j}}}{\Gamma\left(j-\alpha_{j}+1\right)} \frac{C_{2}^{*}}{n^{\sigma-j-1}} \omega_{m-\sigma+1}\left(f^{(\sigma-1)}, \frac{1}{n}\right)= \\
\rho_{n}\left(1-\frac{(x+1)^{h-\alpha_{h}}}{\Gamma\left(h-\alpha_{h}+1\right)}\right)=\rho_{n}\left(\frac{\Gamma\left(h-\alpha_{h}+1\right)-(x+1)^{h-\alpha_{h}}}{\Gamma\left(h-\alpha_{h}+1\right)}\right) \leq \\
\rho_{n}\left(\frac{1-(x+1)^{h-\alpha_{h}}}{\Gamma\left(h-\alpha_{h}+1\right)}\right) \leq 0,
\end{gathered}
$$

and hence again $L^{*}\left(Q_{n}(x)\right) \geq 0, x \in[0,1]$. 


\section{References}

[1] G.A. Anastassiou, Spline monotone approximation with linear differential operators, Approximation Theory and its Applications, Vol. 5 (4) (1989), 61-67.

[2] R. De Vore, G. Lorentz, Constructive Approximation, Springer-Verlag, Heidelberg, New York, 1993.

[3] K. Diethelm, The Analysis of Fractional Differential Equations, Lecture Notes in Mathematics, Vol. 2004, 1st edition, Springer, New York, Heidelberg, 2010.

[4] L.L. Schumaker, Spline functions: Basic Theory, John Wiley and Sons, Inc., New York, 1981. 